\title{
Exploration of the algorithm of automatic recognition of defects in ultrasonic testing image
}

\author{
Xifeng Zhou ${ }^{\mathrm{a}}$, Hao Zou ${ }^{\mathrm{b}}$, Qiangang Guo ${ }^{\mathrm{c}}$ \\ School of Automation, Nanjing University of Posts and Telecommunications, Nanjing, China \\ axifeng@njupt.edu.cn, b838370374@qq.com, 'cgqg810@126.com
}

Keywords: digital image; k-means; projection algorithm; false alarm; defect recognition.

\begin{abstract}
Traditional detection is through a lot of human judgment for defect recognition. The efficiency is low and the accuracy is not high in this way. With the development of digital image processing technology in the industrial filed. Research of automatic recognition on defect image becomes meaningful. In this paper, we puts forward an automatic recognition algorithm on the A defect image sequence. After analyzing the defect image, first of all, we apply k-means cluster segmentation on the original image to get the acoustic images with false alarm. In order to get full acoustic images by preventing the false alarm, we use the projection algorithm and achieved good results. Finally we do the bottom wave and defect wave detection on the acoustic image, and realize the recognition of defect automatically. The experimental results show that the method we proposed has high accuracy.
\end{abstract}

\section{Introduction}

With the development of digital image processing technology in the field of industry, application of image detection based on digital image processing technology is becoming more and more widely. The traditional detection on testing graph is judged one by one through a lot of human ${ }^{[1]}$, the efficiency is low and then the accuracy is not high. So image detection based on digital image processing technology becomes significant. Image automatic identification based on image detection can avoid the process of human judging result one by one of detection in graph based on the tens of thousands of flaw detection. Using the computer record results can save a lot of time and improve the efficiency.

On the basis of domestic and oversea ultrasonic detection, ${ }^{[2]}$ this paper combined with the detection objects-Chinese wheel structure and defect distribution characteristics, we analyze the feature of acquired image and prose the automatic identification algorithm based on image segmentation, virtual elimination and morphological image processing. Experiments show that the method in this dissertation has higher accuracy and efficiency compared with other segmentation algorithms. It can realize the automatic recognition of an image and achieve the expected goal.

\section{Method overview}

Figure 1 shows the basic process of this method, the method in this paper is mainly divided into two basic steps: sonic extraction and defect detection. In the phase of acoustic extraction, our goal is to be able to effectively extract to the testing image in acoustic information. Firstly, we transform the original graph into gray scale, then calculate and analyze the gray scale with histograms.Finally we propose to use the K-means of clustering segmentation to obtain the acoustic with a false alarm. In the phase of defect detection, our object is to get complete acoustic information. We use the horizontal projection and vertical projection algorithm to remove the false alarm, get the full acoustic wave. Finally we make the detection of bottom wave and defect wave and implement the automatic recognition of defect. 


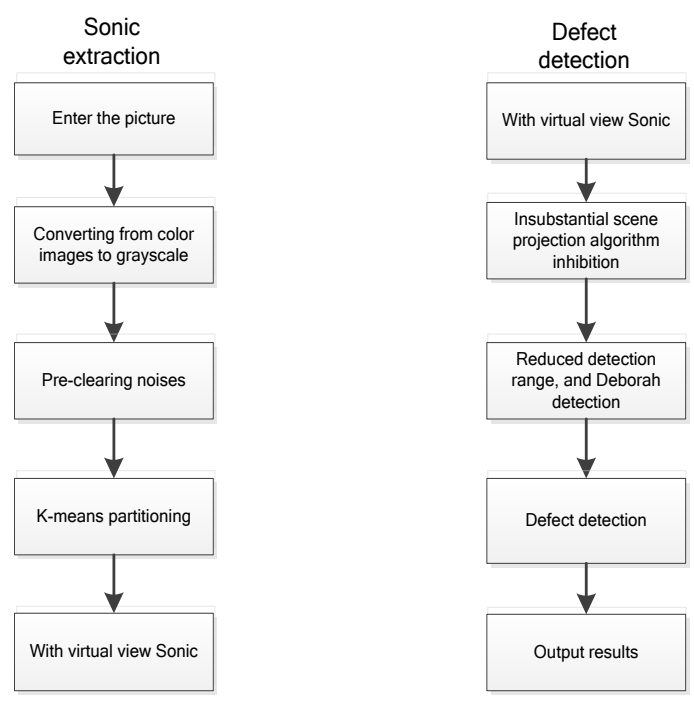

Fig. 1 flow chart of this method

\section{Ultrasonic detection technologies}

Analysis of ultrasonic echo signal. Ultrasonic detection refers to the interaction of ultrasonic and test block, studied on the reflection, transmission and scattering wave, detection of macro defects detection, geometric characteristic measurement, the structure and the mechanical properties of the test block changes, and to the technology of evaluating its application ${ }^{[3]}$. The ultrasonic echo display principle as shown below:

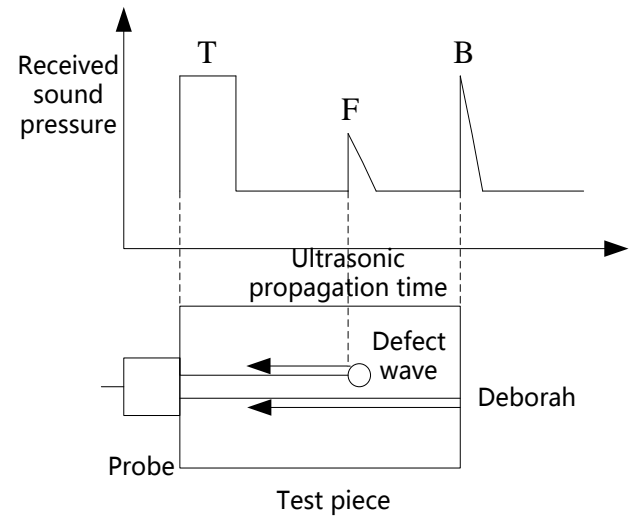

Figure 2 ultrasonic echo display principle

Figure $2 \mathrm{~T}$ represents the beginning wave, $\mathrm{F}$ represents defect wave, $\mathrm{B}$ represents the bottom wave, we can see by ultrasonic echo principle of display: if there is defect wave in front of the ultrasonic just in the bottom crest, then the test block has defects.

\section{Sonic extractions}

K-means clustering segmentation. The process of $k$-means clustering segmentation is described as: we take all the pixels which input into the image as a data set $\left\{x_{1}, \ldots, x_{N}\right\}, \mu_{k}$ represents the center of $\mathrm{k}$ class for every data $x_{n}$, we introduce a two-dimensional indicator variable $r_{n k} \in\{0,1\}$, among of them $k=1, \ldots, K, r_{n k}$ means that if data $x_{n}$ belong to k class, $r_{n k}=1$,or $r_{n k}=0$, it is;

$r_{n k}=\left\{\begin{array}{l}1, \text { if } k=\arg \min _{j}\left\|x_{n}-\mu_{j}\right\|^{2} \\ 0, \text { otherwise. }\end{array}\right.$

Then the deviation function of the data set is defined as;

$$
J=\sum_{n=1}^{N} \sum_{k=1}^{K} r_{n k}\left\|x_{n}-\mu_{k}\right\|^{2}
$$


We learn partial derivative of deviation function on $\mu_{k}$, order partial derivative equal 0 , we learn the expression of $\mu_{k}$, that is;

$$
\mu_{k}=\frac{\sum_{n} r_{n k} x_{n}}{\sum_{n} r_{n k}}
$$

Adopt EM algorithm iterate until the value of deviation function no longer change, then we can obtain the optimum $\mu_{k}$ and $r_{n k}$. The steps of EM algorithm are described as follow

The first step: initialize $\mu_{k}, k=1,2, \ldots, K$

The second step: it is the process of E, keep $\mu_{k}$ unchanged, compute $r_{n k}$ according to formula (2), to lower $\mathrm{J}$.

The third step: it is the process of $\mathrm{M}$, keep $r_{n k}$ unchanged, update $\mu_{k}$ according to formula (4), to lower J.

The fourth step: judge the change of $\mathrm{J}$ whether meet the requirement, if it meets, end of the iteration, or return to the second step and continue the iteration.

\section{Defect detecting}

Projection algorithm eliminate virtual scene. The last section not only detected the sonic wave but also found some false alarms. This part presents an efficient scheme to reject false alarm, through the analysis of virtual scene graph, we can learn that virtual scene are mainly straight-lines continuum, these lines can measure the acoustic wavelength and amplitude. So we can take the horizontal projection and vertical projection algorithm respectively. Count the number of white pixels in the horizontal direction and vertical direction respectively. We set a threshold according to the length and width of the image, then we can eliminate these virtual scene. Finally we can achieve the complete acoustic image. Results are shown as follow ${ }^{[4]}$ : (a) is the gray scale after conversion, (b) is the acoustic image after K-means segmentation, (c) is the acoustic image after eliminating virtual scene.

(a)

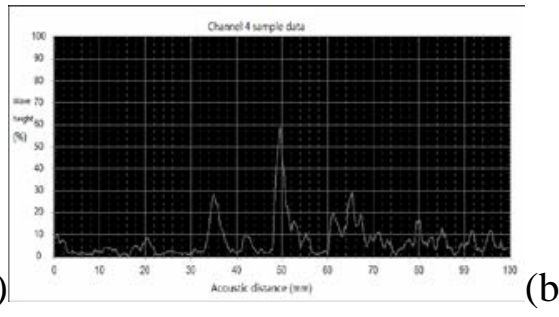

(b)

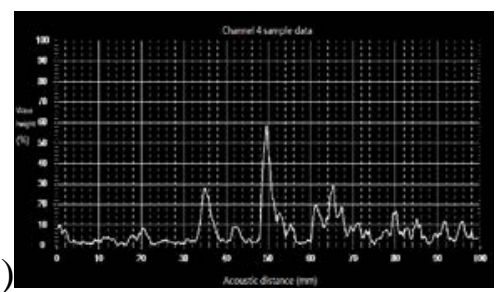

Figure 3 a virtual scene culling

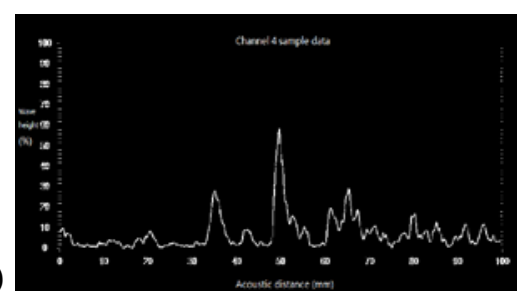

(c)

Defect detection. In the last section, we successfully detected the acoustic wave. Then we will detect the defect wave. According to the characteristics of ultrasonic imaging, our detection is divided into two steps: the bottom wave inspection and defect wave detection. To the detection of bottom wave and defect wave, we can limit the detection range according to the horizontal and vertical coordinates of the white line in the virtual scene. Then detect the peak. On the peak, we count the number of white bright spot in a $5 * 5$ search space [5], with this account to judge the existence of wave peak. The detection results are shown as follows: (a) is the bottom wave detection map, and use the white square showing peaks, (b) is the flaw wave detection map, and use the white square display wave peak.

\section{Experiment results}

All the data used in this paper are all the detection of an image from different test blocks, a total of four image sequences ${ }^{[6]}$. For the sake of convenience, these four sequences are named as sequence1, 
sequence2, sequence3 and sequence4 respectively. Among which sequence1 contains 100 frame, sequence 2 contains 100 frame, sequence 3 contains 100 frame, sequence 4 contains 100 frame. The size of image is for $870 * 410$.

(a)
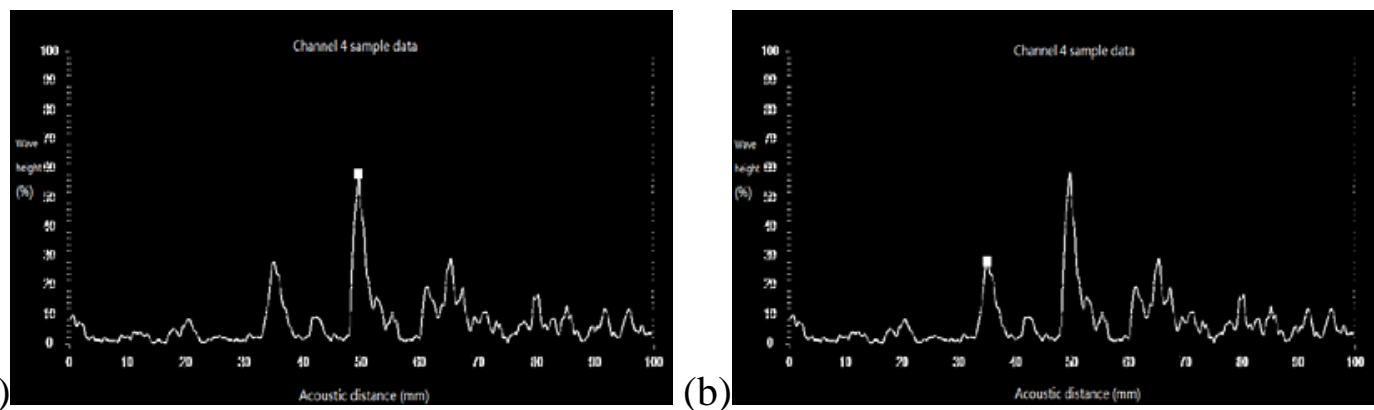

Figure 4 wave nondestructive testing

Statistics on the entire sequence of experiment results are shown as tablet1, we can see from the table that the method we proposed in this paper performs accurately in the aspect of defect detection.

Table. 1 test results

\begin{tabular}{|c|c|}
\hline \multirow{2}{*}{ Name } & The method in this paper \\
\cline { 2 - 2 } & accuracy \\
\hline Sequence 1 & $99 \%$ \\
\hline Sequence 2 & $99 \%$ \\
\hline Sequence 3 & $100 \%$ \\
\hline Sequence 4 & $100 \%$ \\
\hline
\end{tabular}

\section{The end}

This paper presents an accurate algorithm of automatic recognition of defect based on ultrasonic detection in image. At first, we use K-means clustering segmentation on image to obtain defect acoustic image with virtual scene. Then in order to inhibit false alarm and get the complete acoustic images, we propose the projection algorithm and achieve good results. Finally we detect the bottom wave and defection wave in the obtained image, and realize the automatic identification of defect. Experiments show that the method proposed in this paper has high accuracy, it can realize the defect automatic recognition and achieve the expected goal.

\section{References}

[1] Haiyan Fu. The strip surface defect classification and detection and recognition method based on [D] texture analysis. Master Thesis of Northeastern University, 2004

[2] Bao Sheng hou. An image edge detection method based on mathematical morphology [J]. Journal of modern electronic technology, 2010 (8): p.45-46.

[3] Gonzalez. Digital image processing (2nd Ed) [M].Electronic industry press, 2010.7, pp: 496, pp: 427, p: 423-425.

[4] Kernel k-means, Spectral Clustering and Normalized Cut by Intermit S and Brian Kulis.

[5] Nobuyuki Otsu. A Threshold Selection Method from Gray-Level Histogram [J]. IEEE Transactions on Systems and Cybernetics, Vol.SMC-9(1), 2010, p: 62-66.

[6] Ge Wen, the wavelet transform fusion and morphological edge detection algorithm based on [J], Journal of Northeastern University(NATURAL SCIENCE EDITION), twenty-ninth volume fourth issue, 2008 April. 